\title{
STUDY OF INTERDEPENDENCY BETWEEN THE HSDPA AIR INTERFACE AND THE RADIO ACCESS NETWORK
}

\author{
Xinzhi Yan \\ University of Newcastle \\ Newcastle, Australia
}

\author{
Jamil Y. Khan \\ University of Newcastle \\ Newcastle, Australia
}

\author{
Brendan Jones \\ SingTel OPTUS \\ Sydney, Australia
}

\section{ABSTRACT}

This paper analyzes the interdependence between the $\mathrm{I}_{\mathrm{ub}}$ link characteristics and the HSDPA air interface performance using analytical and simulation techniques. The impact of the Node-B scheduling mechanisms are also investigated in this paper. A Markov chain based analytical model and a simulation model is used to obtain HSDPA air interface performance results.

\section{INTRODUCTION}

As one of the initial steps of 3GPP Long Term Evolution (LTE) plan, the release 5 of 3GPP introduced the HSDPA (High Speed Downlink Packet Access) standard to enhance downlink peak data rates, spectral efficiency, and quality of service (QoS) control for packet based services [1]. The HSDPA shared channel uses a short $2 \mathrm{~ms}$ transmission time interval (TTI), and uses variable coding and modulation techniques to achieve high data rates. In addition to above techniques the Node-B based fast scheduling and fast physical layer hybrid automatic repeat request (H-ARQ) techniques are also used. According to the HSDPA roadmap the air interface data rates could reach up to $14.4 \mathrm{Mbps}$ within the next few years. The peak data rate requires extremely favourable radio conditions. It is unlikely that all users in a cell would be able to get such a high data rate, even if only one user was using the cell at a time. In a HSDPA network user data rate will vary with transmission conditions and also due to time and code sharing amongst users. Since average traffic on the $I_{u b}$ link is usually less than the peak rate over the air interface, it is not economical or efficient to reserve $I_{u b}$ link bandwidth at the potential peak rate for the duration of a call. The overall HSDPA throughput and QoS will depend on the air interface and radio link performance. The radio access network can significantly influence air interface performance. The HSDPA architecture incorporates a downlink buffer and scheduler in the Node-B. A flow controller is generally employed on the $\mathrm{I}_{\mathrm{ub}}$ link between a radio network controller (RNC) and a Node-B to avoid buffer overflow or buffer starvation. If an $\mathrm{I}_{\mathrm{ub}}$ link cannot provide high enough instantaneous bandwidth, sufficient number of packets may not be supplied to the Node-B from the RNC to match the service rate of the downlink buffer. In such an event it is possible that the buffer might run out of queued packets and the peak transmission rate over the air interface cannot be sustained [2].

The Node-B based scheduler can take advantage of instantaneous channel variations to maximize the network throughput while satisfying users' QoS requirements [3]. By employing different radio resource management (RRM) algorithms, the scheduler can be configured to provide users with different peak to average ratios of data rates over the air interface. The Round Robin (RR) is simple algorithm, which serves users in a cyclic order ignoring the channel quality conditions and ensures a fair resource distribution among the users. The maximum carrier-to-interference ratio (Max-C/I) serves the user with largest instantaneous supportable data rate to maximize cell throughput. However the main drawback is that a number of users at cell edges may not receive transmission opportunities. The proportional fair (PF) provides a trade-off between fairness and achievable cell throughput [2], [3]. The scheduling algorithm and the $\mathrm{I}_{\mathrm{ub}}$ link characteristics could affect the HSDPA air interface performance. Hence, it is the major objective of this paper to study the impact of $I_{u b}$ link capacity on the HSDPA air interface for different Node-B schedulers. The interaction between the $I_{u b}$ link and the HSDPA air interface is investigated using both analytical and simulation models.

Reviewing the literature in this area, we found several works which have concentrated on the $I_{u b}$ flow control and efficiency analysis for the HSDPA. Legg presented a strategy for the $I_{u b}$ link flow control, which found the scheduler in Node-B to have a major influence on the amount of memory used at the Node-B [4]. Necker et. al. investigated the impact of the $\mathrm{I}_{\mathrm{ub}}$ flow control on the HSDPA system performance and correlated larger protocol delay and resource grant update period between the Node-B and the RNC with a deterioration in IP packet delay characteristics [5]. Toskala et. al. studied the $I_{u b}$ link efficiency on the HSDPA air interface [6]. None of the previous works investigated the interdependencies between the RAN and the air interface.

This paper is organized as follows. The HSDPA protocol architecture is briefly introduced in section II. Section III describes a flow control algorithm and its corresponding Markov chain model. The $\mathrm{I}_{\mathrm{ub}}$ based transport techniques and simulation models are introduced in the section IV. Simulation results are presented in the section V. Section VI presents the conclusions.

\section{HSDPA PRotocol ARCHITECTURE}

The HSDPA user plane protocol architecture is shown in Fig.1. The Radio Link Control (RLC) layer handles the segmentation and retransmission for user and control data. The MAC-d (dedicated medium access control) layer is retained in RNC to handle transport channel switching. The MAC-hs (hs for high speed) is located in the Node-B and is responsible for scheduling, priority handling, H-ARQ, and selection of an appropriate transport format and resource etc. The HS-DSCH (High Speed Dedicated Shared Channel) is the transport channel that carries the actual user HSDPA data. The HS-DSCH FP (Frame Protocol) handles the data transport between a RNC and a Node-B. For a HSDPA connection data received from a core network is handled by 
RLC and MAC-d layers creating MAC-d PDUs (Protocol Data Unit) which is then packed into a HS-DSCH frame, and transmitted on the $\mathrm{I}_{\mathrm{ub}}$ link.

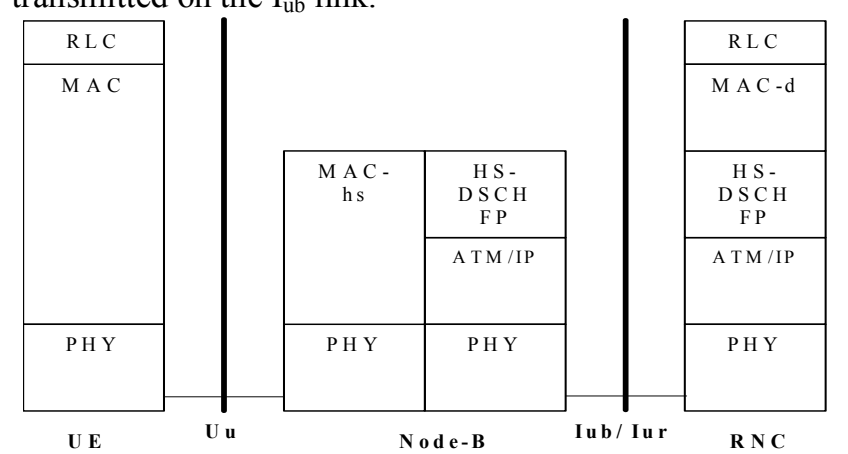

Figure 1. HSDPA protocol architecture.

\section{I UB $_{\text {B }}$ Flow CONTROL AlgORITHM}

The flow control is an important technique used in packet switched networks to reduce the likelihood of buffer overflow and to improve link utilization. In a recently published 3GPP technical report the E-UTRAN architecture specifies the transmission delay between UE (User Equipment) and RNC should be lower than $10 \mathrm{~ms}$ [7], [8]. The delay limit on the downlink is expected to be even shorter than the uplink. To ensure a lower transmission delay between the RNC and the UE on the downlink it is necessary to employ a flow control algorithm between the two nodes. Fig. 2 shows the basic operation of the proposed flow control algorithm. As shown in the figure, the RNC maintains a large buffer of packets which is updated via the core network. The Node-B maintains a minimum buffer size which enables the use of peak transmission rates for HSDPA users on the air interface. The flow control algorithm continuously updates the Node-B buffer based on the HSDPA connection throughput and demands.

In this section we present an analytical model to analyze the performance of the HSDPA air interface using the Markov chain model. For each HSDPA user we allocate a fixed space in the Node-B buffer. We assume that $n$ represents the buffer size for each user in the Node-B buffer, where $n$ corresponds to the total number of MAC-d PDUs that could be sent over the air interface at the peak rate in every TTI ( $2 \mathrm{~ms})$ under the best transmission channel condition. We assume that the $\mathrm{I}_{\mathrm{ub}}$ link bandwidth is $m$, where $m$ is the number of MAC-d PDUs sent in each TTI over the link. After each air-interface transmission event on the air interface, the Node-B immediately requests the RNC to send more MAC-d PDUs to replenish its buffer. The request size $R_{s}$ in number of PDUs is equal to the free buffer size. If the requested size is larger than the $\mathrm{I}_{\mathrm{ub}}$ bandwidth $m$, i.e. $R_{s}>m$, then only $m$ MACPDUs are delivered to the Node-B since attempting to deliver a larger value would lead to $\mathrm{I}_{\mathrm{ub}}$ link congestion and even greater delay in refilling the buffer. The value of $R_{s}$ could vary between 1 to $n$. To avoid low $\mathrm{I}_{\mathrm{ub}}$ link utilization we maintain the following relationship $m<n$. Utilising the above flow control algorithm the Node-B buffer is used to adjust the HSDPA user throughput on the air interface based on the radio link condition. It is possible that a peak air interface transmission rate may not be sustainable due to $\mathrm{I}_{\mathrm{ub}}$ link and the air interface transmission rate mismatch. Readers are reminded that the air interface throughput is radio channel condition dependent and varies over time.

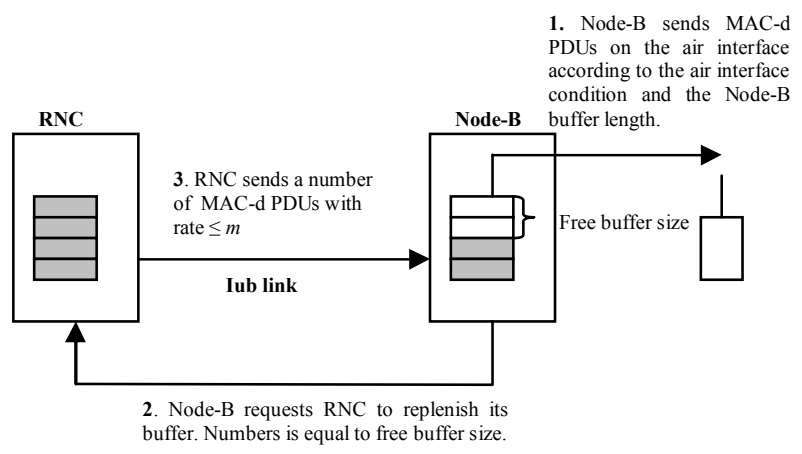

Figure 2. flow control between Node-B and RNC

We now present a brief analysis of HSDPA transmission channel efficiency. We define HSDPA transmission channel efficiency $\eta$ as the ratio of the actual number of MAC PDUs available for transmission in the Node-B buffer to the maximum number of MAC PDUs that can be carried by the air interface condition per TTI, as shown in equation (1). The HSDPA transmission efficiency takes into account the radio channel condition, buffer length, and the $\mathrm{I}_{\mathrm{ub}}$ link effective bandwidth. For example, on one occasion, if 8 PDUs of a HSDPA connection is available in the Node-B buffer but the radio link condition permits transmission of 10 PDUs then we obtain a $\eta$ value of $0.8(8 / 10)$. In that case when a transmission opportunity arises the Node-B cannot transmit the maximum number of PDUs. This situation can be avoided by increasing the fixed allocation of bandwidth on the $\mathrm{I}_{\mathrm{ub}}$ link but that will reduce the $\mathrm{I}_{\mathrm{ub}}$ link efficiency.

$$
\eta=\frac{\text { Actual_}_{-} M A C_{-} P D U_{-} T x}{M a x_{-} M A C_{-} P D U_{-} a i r}
$$

Due to the time-variant nature of radio channels, the transmission data rate of the air interface is highly variable. We assume that the transmission rate (expressed in number of MAC-d PDUs per TTI) of a HSDPA connection is distributed between 0 and a maximum number $n$. The value of $n$ depends on the UE category. Let $r$ represent the air interface rate, which the radio channel condition can support in terms of PDU. Let $R_{i}$ represents the probability of air interface transmission rate, where $i$ represent the number of PDUs transmitted at that rate. After the Node-B transmits PDUs on the air interface, it is assumed that the Node-B immediately requests the $\mathrm{RNC}$ to send packets to replenish its buffer before the next transmission opportunity. Let $l_{n}$ be the number of MAC-d PDUs in the Node-B buffer measured after the buffer is replenished and the next transmission opportunity over the air interface in the $n$-th TTI is pending. The buffer length could vary between $m$ and $n$, i.e. $m \leq l_{n} \leq n$. We can form a Markov chain using the values of $l_{n}$. Consider the stationary state where all $\left\{l_{n}\right\}$ have the same probability distribution. Let $l$ denote the random variable in MAC-d PDU 
numbers in the Node-B buffer. The transition matrix is shown in equation (3).

Let, $\pi_{i}=P\{l=m+i\}, \quad i=0, \ldots ., n-m$

The transition matrix $\mathrm{P}$ is shown below.

$$
\left(\begin{array}{ccccc}
\sum_{i=m}^{n} R_{i} & R_{m-1} & \ldots & R_{2 m-n+1} & \sum_{i=0}^{2 m-n} R_{i} \\
\sum_{i=m+1}^{n} R_{i} & \ddots & \sum_{i=0}^{2 m-n+1} R_{i} \\
\vdots & & & \vdots \\
R_{n} & R_{n-1} \cdots & R_{n-m-1} & \sum_{i=0}^{m} R_{i}
\end{array}\right) \quad(m \leq n \leq 2 m)
$$

In the stationary state, we can write $\pi=\pi P$, so

$$
\begin{aligned}
& \pi_{0}=\pi_{0} \sum_{i=m}^{n} R_{i}+\pi_{1} \sum_{i=m+1}^{n} R_{i}+\ldots+\pi_{n-m} R_{n} \\
& \pi_{1}=\pi_{0} R_{m-1}+\pi_{1} R_{m}+\ldots .+\pi_{n-m} R_{n-1} \\
& \vdots \\
& \pi_{n-m-1}=\pi_{0} R_{2 m-n+1}+\pi_{1} R_{2 m-n}+\ldots .+\pi_{n-m} R_{n-m-1} \\
& \pi_{n-m}=\pi_{0} \sum_{i=0}^{2 m-n} R_{i}+\pi_{1} \sum_{i=0}^{2 m-n+1} R_{i}+\ldots .+\pi_{n-m} \sum_{i=0}^{m} R_{i}
\end{aligned}
$$

$$
\pi_{0}+\pi_{1}+\cdots+\pi_{n-m}=1
$$

Recalling the HSDPA link efficiency definition as shown in the equation (1), we then calculate HSDPA transmission efficiency.

$$
\begin{aligned}
& \text { When } l=m, \\
& \text { if } r \leq m, \eta_{l=m}=100 \% \text {; } \\
& \text { if } r=m+1, \eta_{l=m}=\frac{m}{m+1} \text {; } \\
& \vdots \\
& \text { if } r=n, \eta_{l=m}=\frac{m}{n} \text {; } \\
& \vdots \\
& \text { When } l=n-1, \\
& \text { if } r \leq n-1, \eta_{l=n-1}=100 \% \text {; } \\
& \text { if } r=n, \eta_{l=n-1}=\frac{n-1}{n} \text {; }
\end{aligned}
$$

When $l=n$,

$$
\eta_{l=n}=100 \% \text {; }
$$

So, the average HSDPA transmission efficiency is given by Equation (5):

$$
\begin{aligned}
\eta= & \pi_{0}\left(\sum_{i=0}^{m} R_{i} \times 1+R_{m+1} \times \frac{m}{m+1}+\ldots .+R_{n} \times \frac{m}{n}\right)+ \\
& \pi_{1}\left(\sum_{i=0}^{m+1} R_{i} \times 1+R_{m+2} \times \frac{m+1}{m+2}+\ldots .+R_{n} \times \frac{m+1}{n}\right) \\
& +\ldots .+\pi_{n-m} \times 1
\end{aligned}
$$

We use a single HSDPA cell, which supports category 12 UEs, as an example to analyze the relationship between the HSDPA transmission efficiency and the $I_{u b}$ bandwidth. A fixed RLC/MAC PDU size of 320bits is used and the maximum number of RLC/MAC PDU is set to 10 .

From the statistical point of view, the air interface capacity of a cell will be approximately normally distributed according to the Central Limit Theorem. Since the distribution of air interface rate is discrete, to simplify our analysis, we approximate the HSDPA air interface data rate using the following binomial distribution (6).

$$
\begin{aligned}
& R_{i}=\left(\frac{n}{i}\right) p^{i}(1-p)^{n-i} \quad 0<i \leq n ; \quad 0<p \leq 1 \\
& \text { mean }=n \cdot p \\
& \text { HSDPA average throughput at RLC layer } \\
& =n \times p \times \text { RLC_Packet_size/TTI }
\end{aligned}
$$

Where $R_{i}$ represents the probability of transmission rate air interface support, $i$ represents the number of PDUs transmitted at that rate, and $n$ is equal to the maximum number of MAC-d PDUs that can be sent in every TTI. The value of $p$ depends on cell parameters. We assume that a very strong and controlled coverage in a small cell and low user mobility could lead to higher value of $p$, which represents higher air interface transmission rate for users. Best radio channel condition is represented by the value of $p=1$. Fig. 3 shows the probability distribution of air interface transmission rate for $p=0.7$, and $n=10$ for a category $12 \mathrm{UE}$. This plot shows that for $p=0.7$ a HSDPA link may transmit a number of PDUs per TTI at different times; there is a high probability that a UE will transmit 6 to 8 PDUs per TTI. The peak of the plot will shift towards left for lower values of $p$. Similar results were reported by an Ericsson research group that measured HSDPA network performance in different transmission conditions [9].

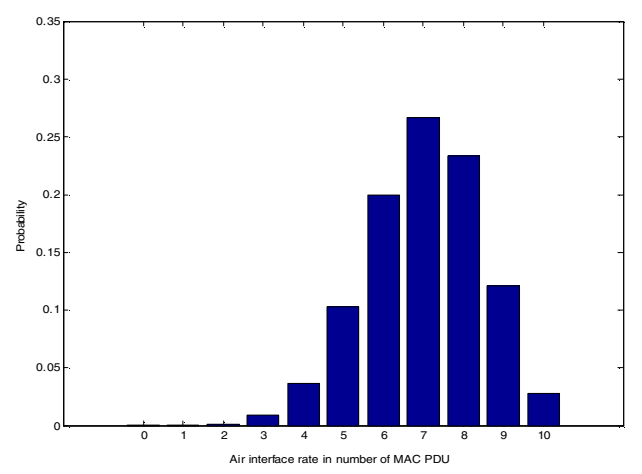

Figure 3. Probability distribution of air interface represented in number of MAC-d PDUs per TTI for $\mathrm{p}=0.7$ and $\mathrm{n}=10$.

Using the above analytical technique, we can calculate the HSDPA air interface efficiency for a category 12 UE with different air interface conditions and $\mathrm{I}_{\mathrm{ub}}$ link capacities. Analytical results are shown in Fig.4. The figure shows that the value of $\eta$ increases with the $\mathrm{I}_{\mathrm{ub}}$ data rate. The figure also shows that the degree of improvement depends on the air interface condition. In a realistic HSDPA network the air interface throughput will vary depending on the transmission channel condition. Hence, a fixed $\mathrm{I}_{\mathrm{ub}}$ bandwidth allocation 
will either reduce the air interface efficiency or it will reduce the $\mathrm{I}_{\mathrm{ub}}$ link utilization.

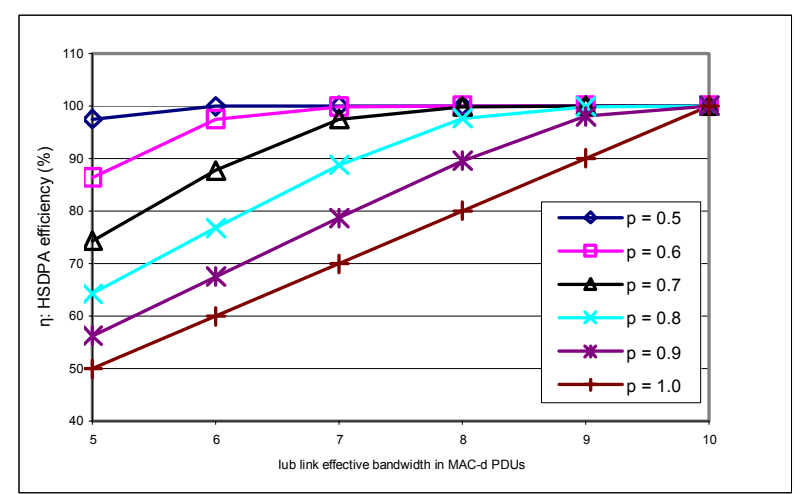

Figure 4. The relationship between the air interface efficiency and the $\mathrm{I}_{\mathrm{ub}}$ link bandwidth under different air interface conditions.

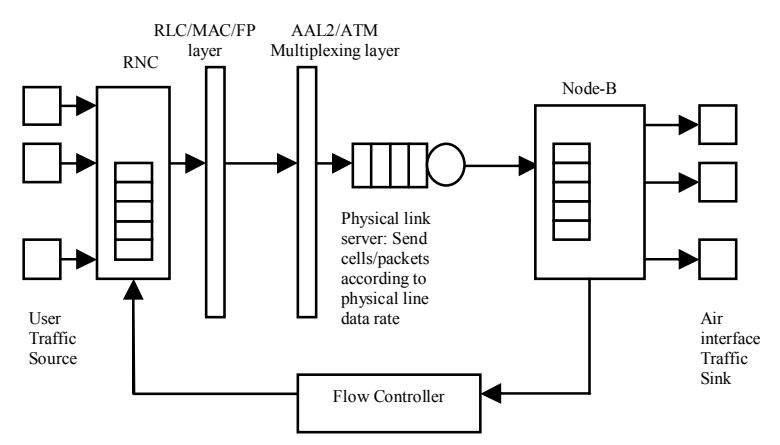

Figure 5. The simlator structure

\section{Simulation Model}

A simulation model has been developed to analyze the effect of the $I_{u b}$ link characteristics on HSDPA transmission efficiency. In the following sections we briefly describe our simulator structure.

As shown in Fig. 5, the simulator consists of an $I_{u b}$ interface and a physical link, a traffic generator, an air interface link and the proposed flow controller. We assume that the Core Network (CN) can deliver enough packets to the RNC so that sufficient packets are queued in the RNC buffer during the active period of a data burst. The flow controller is implemented according to the description of the section III. The Node-B allocates each HSDPA connection a transmission bandwidth according to the radio channel condition which is simulated by the binomial distribution as shown in the Fig. 3. Three scheduling algorithms RR, Max$\mathrm{CQI}$ and PF are separately used at Node-B. In the model the air interface actions were simulated by traffic sinks as shown on the right side of the figure. These traffic sinks simulate variable radio channel conditions. On the RNC side the RLC/MAC layer headers are added to form MAC PDUs, and then packed into a FP frame. A FP frame is segmented into a number of AAL2/ATM cells. At the Node-B RLC/MAC PDUs are extracted and stored in the Node-B buffer for transmission on the downlink [10].

\section{Simulation Results}

In this section several simulation results are presented which are obtained using the simulation model. Simulation results are also compared with analytical results obtained using the Markov model. Fig. 6 shows the relationship between the value of $p$ (air interface channel condition) and the HSDPA efficiency $\eta$ for category $5 / 6$ and category 12 UEs. Comparing the analytical and simulation results we find both results are in very close agreement. The figure shows that the value of $\eta$ decreases as the transmission channel quality improves because the $I_{u b}$ link creates the bottleneck. In this simulation for category 12 we used a 2.048 Mbps AAL2/ATM based $\mathrm{I}_{\mathrm{ub}}$ link which supports 8 MAC PDUs per TTI transmission between the RNC and the Node-B. For category 5/6 link rate of $4.096 \mathrm{Mbps}$ were used. Fig. 6 shows that both category connections behaves similarly however, the category $5 / 6$ connections offer slightly better performance because of use of higher $\mathrm{I}_{\mathrm{ub}}$ link rate. Next we observe the relationship between the transmission channel condition and the combined air interface and $\mathrm{I}_{\mathrm{ub}}$ link throughputs. Fig. 7 shows that the HSDPA air interface throughput can linearly increase with the increasing value of $p$. However, due to the $\mathrm{I}_{\mathrm{ub}}$ link constraints, the air interface throughput remains limited at 1.3 Mbps for a 2.048 Mbps $\mathrm{I}_{\mathrm{ub}}$ link based on the AAL2/ATM transport protocol. On the other hand the graph also shows that the $\mathrm{I}_{\mathrm{ub}}$ link utilization remains low for lower values of $p$ because of lack of demand by the air interface. In this case if a high bandwidth $\mathrm{I}_{\mathrm{ub}}$ link is used then for average transmission channel conditions the $\mathrm{I}_{\mathrm{ub}}$ link utilization will drop. So, these results show that simply increasing the $\mathrm{I}_{\mathrm{ub}}$ link capacity is not sufficient to increase the overall HSDPA link efficiency. Further we examine the Node-B buffer occupancy levels for different air interface transmission conditions as shown in Fig. 8. The figure shows both analytical and simulation results. The figure clearly shows that they are in very close agreement. The graph shows that for the $p$ value of 0.7 the buffer occupancy level is 10 MAC PDU for about $80 \%$ of the time whereas for $p=0.9$ for about $90 \%$ time the buffer length is 8 the minimum buffer size. The reason for shorter buffer length for $p=0.9$ is that the Node-B drain rate by the air interface is higher than the buffer replenishment rate by the $\mathrm{I}_{\mathrm{ub}}$ link. The buffer length is measured after every transmission on the air interface and its subsequent buffer replenishment by the $\mathrm{RNC}$. The graph shows that $p=0.8$ is a balanced region where drain rate is matched by the replenishment rate.

Next, we analyze the impact of Node-B scheduling algorithms on HSDPA performance. In the simulation, we use total 10 category 12 users. A $2.048 \mathrm{Mbps}$ AAL2/ATM based $\mathrm{I}_{\mathrm{ub}}$ link is used. The $p$ value for air interface conditions of each UE is controlled by a uniform distribution where values could vary between 0.5 to 1.0 . Fig. 9 shows the cumulative distribution function (CDF) of potential air interface throughput and the actual delivered throughput using RR, Max-CQI and PF scheduling algorithms. The Max-CQI fully exploits the available capacity thus offering the highest potential HSDPA throughput. The RR offers a low potential throughput, because it ignores the air channel quality, and 
there is no multi-user diversity gain for this algorithm. The PF provides a trade-off between fairness and achievable cell throughput. In this case modest throughput gain is achieved comparing to the RR. Due to $\mathrm{I}_{\mathrm{ub}}$ link capacity constraint, the actual delivered HSDPA throughputs are lower than potential ones for a good radio channel condition. Fig. 10 shows the HSDPA efficiency for different scheduling algorithms. Results indicate the impact of $\mathrm{I}_{\mathrm{ub}}$ link capacity constraint is quite significant when the Max-CQI scheduler is used. In this case the $\mathrm{I}_{\mathrm{ub}}$ link could become the main bottleneck.

\section{CONCLUSIONS}

In this paper we presented novel analytical and simulation techniques to study the relationships between the HSDPA air interface and $\mathrm{I}_{\mathrm{ub}}$ link characteristics. The scheduling algorithm used at Node-B is also an important factor when allocating radio access network capacity. For next generation HSDPA services it is necessary to properly dimension radio access networks to support high data rate multimedia services over air interface. The results can be used as a reference for network designers.

\section{REFERENCES}

[1] T. E. Kolding, F. Frederiksen, and P. E. Mogensen, "Performance aspects of WCDMA systems with high speed downlink packet access (HSDPA)," presented at Proceedings. VTC 2002-Fall. 2002.

[2] H. Holma, A. Toskala, HSDPA/HSUPA for UMTS, John Wiley and Sons, 2006, pp.26-27.

[3] P. Jose, "Packet Scheduling And Quality of Service in HSDPA," PhD Thesis, Department of Communication Technology, Aalborg University, 2003

[4] P.J.Legg, "Optimised Iub flow control for UMTS HSDPA" in Proc IEEE VTC 2005-Spring, Stockholm, Sweden, Jun 2005.

[5] M. C. Necker and A. Weber, "Impact of Iub flow control on HSDPA system performance," Proc. PIMRC 2005, Berlin, Germany, September 2005.

[6] A. Toskala, H.Holma, E. Metsala, K. I. Pedersen, and D. Steele, "Iub efficiency analysis for high speed downlink packet access in WCDMA", Proc. WPMC, Aalborg, Denmark, September, 2005.

[7] 3GPP 25.913 "Requirements for Evolved UTRA (E-UTRA) \& Evolved UTRAN (E-UTRAN)", Release 7, v.7.3, 03/2006.

[8] A. Toskala, et. al. "UTRAN Long Term evolution in 3GPP", Proc. PIMRC 2006, Helsinki, Finland, September 2006.

[9] J.Derksen, et. al. "HSDPA performance and evolution", Ericsson Review, Vol.3, 2006.

[10] 3GPP TS 25.308 v6.3.0 (2004-12), "High Speed Downlink Packet Access (HSDPA); Overall description; Stage 2 (Release 6)".

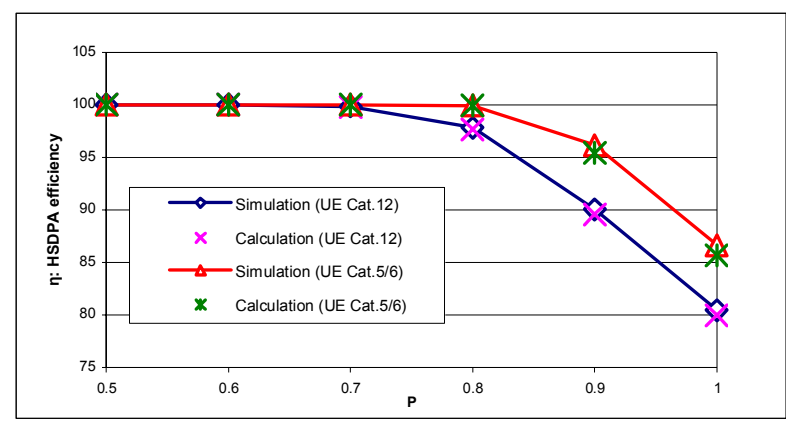

Figure 6. HSDPA efficiency for different values of $p$.

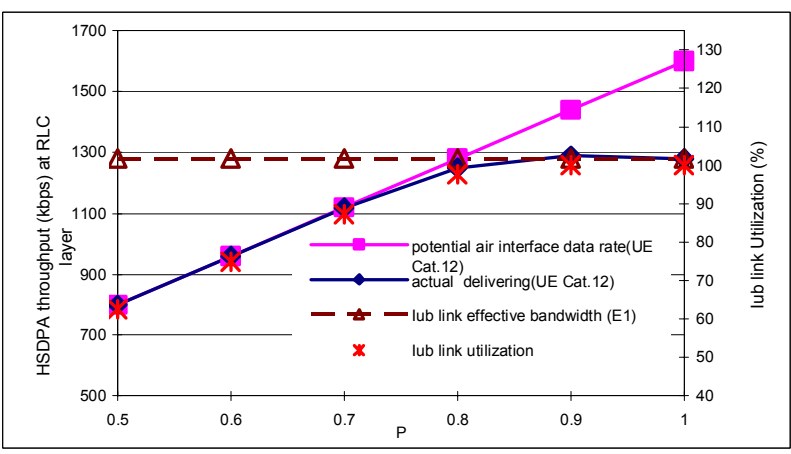

Figure 7. Relationship between the HSDPA average throughput (RLC layer) and $\mathrm{I}_{\mathrm{ub}}$ link utilization

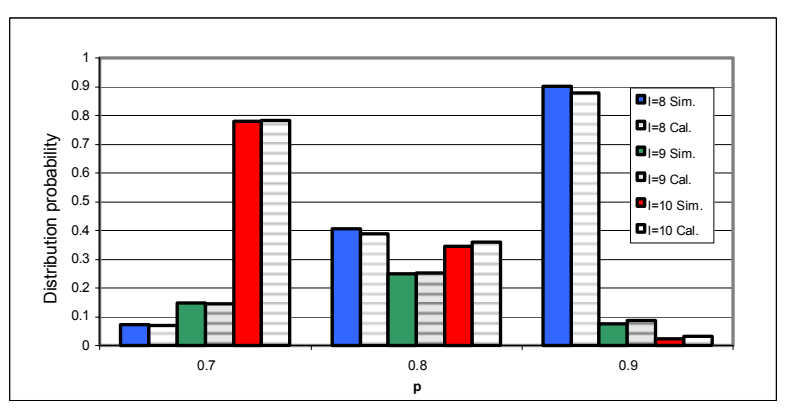

Figure 8. Distribution number of PDUs in the Node-B buffer for UE Cat.12

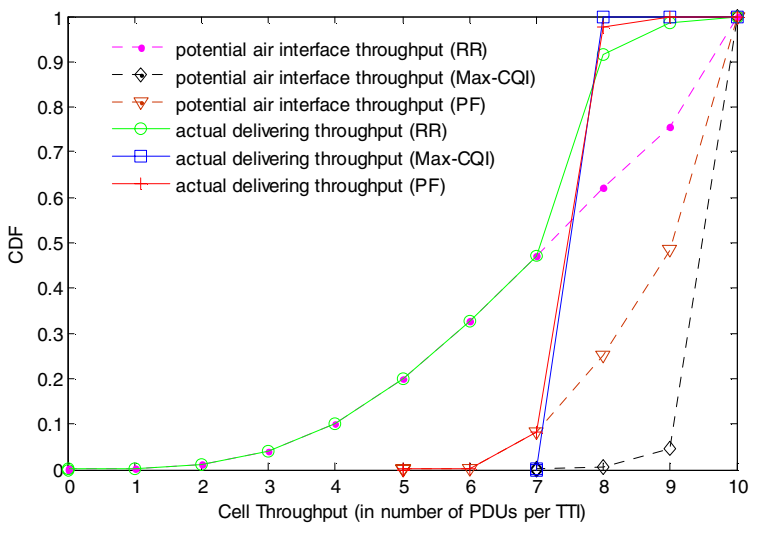

Figure 9. Potential and actual cell throughput

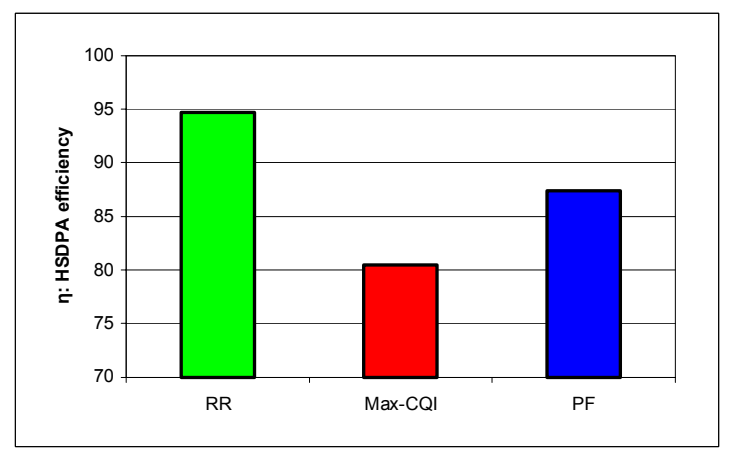

Figure 10. HSDPA efficiency for different scheduling algorithms 